\title{
Study of Polymerization Growth Uniformity in Polymer Gel Dosimeter
}

\author{
A. MEDJAKAL ${ }^{a}$ AND K. MANSOUR ${ }^{a, b, *}$ \\ ${ }^{a}$ Laboratory of Electronic Materials Study and Their Medical Applications, \\ University Brothers Mentouri - Constantine 1, Ain El Bey Road, 25000 Constantine, Algeria \\ ${ }^{b}$ University Salah Boubenider - Constantine 3, \\ University Town Ali Mendjeli, Constantine, Algeria
}

Received: 28.09.2020 \& Accepted: 27.11.2020

Doi: 10.12693/APhysPolA.139.84 *e-mail: karim.mansour@univ-constantine3.dz

\begin{abstract}
In this work, the spatial uniformity of the polymerization growth of the MAGIC gel in the case of an internal irradiation with iodine-131 was studied. A compartmental mathematical model taking into account the kinetics of polymerization of the MAGIC gel was developed and validated by experimental measurements. In order to check the radioactivity homogeneity distribution in the samples, we performed their scintigraphy, while - in order to study the rate of polymerization — we measured the intensity of the MRI signal emitted by each of the samples. The wide dispersion in signal strength observed between different regions of the same sample indicates that the value of the relaxation time T2 varies from one point to another. Scintigraphy and MRI images showed that a homogeneous distribution of ${ }^{131} \mathrm{I}$ activity does not necessarily imply isotropic polymerization. The phenomenon is probably due to the fact that iodine-131 emits beta particles with different energies and the length of polymers does not increase with the same speed for all the polymers in formation. On this basis, we have established a mathematical model which compartmentalizes different species of protons which participate in the modification of the MRI signal.
\end{abstract}

topics: internal dosimetry, MAGIC gel, radio-induced polymerization, mathematical modelling

\section{Introduction}

Internal radiation therapy allows a higher dose of radiation in a smaller area than might be possible with external radiation treatment [1]. However, few dosimetric data are available [2]. Different approaches in radionuclide dosimetry depend on many factors [3-5]. Between the need to document the delivered irradiation and the possibility to optimize the treatment, the development of clinical dosimetry is contrasted. It is now possible to perform personalized dosimetries, considering the pharmacokinetics of radiopharmaceuticals and the morphology of each patient. Knowledge of the absorbed dose and determination of the dose-response relationship for critical organs and tumors are necessary to optimize treatments. Dosimetric tools adapted to this technique are also used because the particles have a weak path in the body and conventional dosimeters do not allow a three-dimensional reconstruction of the distribution of an absorbed dose.

Polymer gel dosimetry is a technique used to verify radiation dose distribution delivered by cancer radiotherapy. The polymerization consists of the addition of monomers and cross-linking of polymer chains induced by free radicals from radiolysis of water. Radiosensitive gels are detectors capable to restore the spatial distribution of the absorbed dose [4]. The quantity of a polymer formed at different locations depends on the amount of radiation received at the particular location.

A new type of polymer gel dosimeters, which respond well to an absorbed dose, manufactured in the presence of normal levels of oxygen, was described by Fong et al. [6] and referred to by the acronym MAGIC (methacrylic and ascorbic acid in gelatin initiated by copper). The response to an absorbed dose can be evaluated using magnetic resonance imaging (MRI). The MRI signal is directly related to the density of protons. However, the intensity of this signal depends on the relaxation time which varies according to the environmental situation of the proton. The relaxation time of a proton belonging to a monomer will be different from that of a proton belonging to a polymer. However, the uncertainty in the measured dose has two sources: the uncertainty due to a calibration curve and the uncertainty from the $\mathrm{R} 2$ map which is the most important [7].

High energy radiation interacts with organic matter by various physical and chemical mechanisms resulting in the formation of short-lived excited 
species and of chemical entities, such as thermalized electrons and neutral or ionic free radicals exhibiting longer life times, allowing them to undergo bimolecular reactions with various molecular compounds by translational diffusion [8].

The aim of this paper is to study the spatial uniformity of the polymerization growth of the MAGIC gel in the case of an internal irradiation by iodine-131. A compartmental mathematical model taking into account the kinetics of polymerization of the MAGIC gel is discussed in Sect. 2 and the experimental results which allowed us to validate this mathematical model are examined in Sect. 3 .

\section{Material and methods}

\subsection{Preparation of samples}

The MAGIC gel was prepared according to the protocol established by Fong et al. [6]. This protocol can be schematized in the following steps:

1. Deionised water in the amount of $700 \mathrm{ml}$ (water for HPLC BIOCHEM Chemopharma) is poured into a beaker under constant agitation to which $80 \mathrm{~g}$ of gelatine (BIOCHEM Chemopharma) are added.

2. The mixture is heated to $50^{\circ} \mathrm{C}$.

3. At $50^{\circ} \mathrm{C}, 2 \mathrm{~g}$ of hydroquinone (BIOCHEM Chemopharma) diluted in $48 \mathrm{ml}$ of deionized water are added.

4. The solution is air-cooled at $38^{\circ} \mathrm{C}$, then $0.35 \mathrm{~g}$ of ascorbic acid (BIOCHEM Chemopharma) diluted in $50 \mathrm{ml}$ of deionized water and $0.02 \mathrm{~g}$ of copper sulfate (SIGMA-ALDRICH) diluted in $30 \mathrm{ml}$ of deionized water are added.

5. The new mixture is shaken for $3 \mathrm{~min}$.

6. Methacrylic acid (BIOCHEM Chemopharma) with a concentration of $9 \%$ is added to the gel which is shaken for $3 \mathrm{~min}$ to make it homogeneous.

The obtained gel is poured into 10 bottles with screw caps and each bottle contains $90 \mathrm{ml}$ of this gel. These vials were vortex-loaded for $2 \mathrm{~min}$ and then refrigerated at $4^{\circ} \mathrm{C}, 18 \mathrm{~h}$ before adding iodine131. In order to verify the density homogeneity of the gel, we checked each of the samples by computed tomography and measured the dispersion of the CT numbers values. The dispersion of the measured CT numbers varies between 2 and $4 \%$ which confirms the homogeneity of the samples. The tomodensitometric images were carried out using a General Electric CT Optima 660 (128 slices - 2015).

To manufacture the amounts of radioactivity to be added to the gel samples, the contents of a $4.0 \mathrm{GBq}$ capsule are diluted in $80 \mathrm{ml}$ of deionized water and activities of 200 to $600 \mathrm{MBq}$ with a $50 \mathrm{MBq}$ step have been formed. The mixture is stirred during $3 \mathrm{~min}$ and then poured into five Petri dishes. Each of them contains $20 \%$ of the added activity. Moreover, we made sure that the Petri dishes were completely filled to prevent residual air from reducing polymerization and causing spontaneous polymerization stripes.

To check the homogeneity of the radioactivity distribution in the gel, scintigraphic images of the Petri dishes containing the samples were made 1 and $3 \mathrm{~h}$ after the preparation of the iodine-131-MAGIC gel. The scintigraphic images were made by a GE Discovery NM/CT670 scanner.

In order to analyze the spatial uniformity of polymerization growth in the MAGIC gel dosimeter, two MRI acquisitions were taken 72 and $120 \mathrm{~h}$ after the preparation of the gel samples. To achieve the thermal balance and a uniform result, the Petri dishes were placed in the treatment room $12 \mathrm{~h}$ before the images were made. Measurements were realised on a GE model 1.5 Tesla MRI imager with a head antenna. The spin echo method was used with the following parameters: the TE (time echo) of $20 \mathrm{~ms}$, the repetition time of $2000 \mathrm{~ms}$ and the matrix size of $256 \times 256$.

\subsection{Mathematical modelling}

The polymerization initiated by ionizing radiation occurs through the mechanism of the radiolysis of water, thus free radicals [9]. The general polymerization process can be broken down into four steps: the formation of free radicals, the initiation, the propagation and the termination [10]. Several advantages can be found in the particularities of a radiation-initiated polymerization which benefit the processing efficiency or produce final materials with unique properties [11].

The chain initiation can take place as soon as the properly selected initiator starts to decompose into free radicals. The initiation is completed when the initiator radical has been added to the first monomer unit, $M$, to produce the chain initiating species $M_{1}^{\bullet}$. From a kinetic standpoint, the rate of initiation $R_{i}$ is controlled by the initiator decomposition. Though directly related to the decomposition rate of the initiator $R_{d}$, the effective initiation rate $R_{i}$ depends on the efficiency factor which takes into account the fraction of generated free radicals that effectively add to the monomer for producing $M_{1}^{\bullet}$ — the chain initiating species [12].

The propagation consists of the iterative growth of the initiating radical $M_{1}^{\bullet}$ that forms, after enough additions, a macro radical. By comparing the effects of substituents on the stability of the free radical, higher propagation rate constants are observed for less stabilized active centers. However, for achieving the formation of chains with a high degree of polymerization, the reactivity of free radicals must be mainly directed towards propagation, limiting competing reactions of a charge transfer [13]. The radiolysis is the radiation-induced dissociation of water molecules in several highly reactive radicals and ions [14]. 
(a)

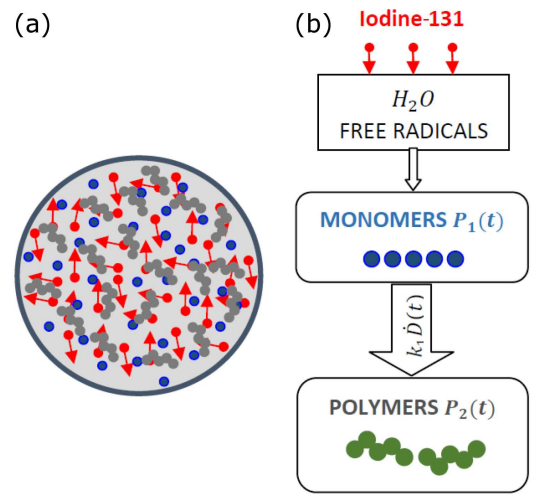

Fig. 1. (a) Schematization of a polymerization process, (b) proposed bicompartmental model.

All steps can be described by mathematical equations. It is important to note that all speeds are time-dependent because the dose rate is also timedependent. Thus, the rate of initiation of chain radicals $R_{i}(t)$ may be written as

$$
R_{i}(t)=\left(\frac{\mathrm{d}\left[M^{\bullet}\right]}{\mathrm{d} t}\right)_{i}=2 f k_{d}[I(t)],
$$

where $\left[M^{\bullet}\right]$ represents the total concentration of all chain radicals irrespective of size and $[I(t)]$ is the initiator concentration at time $t$. The rate constant $k_{d}$ is the rate for its decomposition into two radicals $R^{\bullet}$ per molecule and $f$ represents the fraction of these primary radicals initiating the chains.

Furthermore, the rate of chain termination $R_{t}$ may be written as

$$
R_{t}(t)=-\left(\frac{\mathrm{d}\left[M^{\bullet}\right]}{\mathrm{d} t}\right)_{t}=2 k_{t}\left[M^{\bullet}\right]^{2} .
$$

When $R_{i}(t)=R_{t}(t)$, the steady-state concentration of chain radicals can be expressed by

$$
\left[M^{\bullet}\right]=\sqrt{f \frac{k_{d}}{k_{t}}[I(t)]},
$$

while the rate of propagation is then

$$
R_{p}(t)=k_{p}[M] \sqrt{f \frac{k_{d}}{k_{t}}[I(t)]} .
$$

Note that $R_{p}(t)$ also represents the rate of polymerization.

In the ionizing radiations case, the factor $f$ represents the quantum yield for the chain initiation (the number of pairs of chain radicals generated per quantum absorbed).

To establish a description of the variations in the proton densities of different species contributing to the formation of the MRI signal, we have classified them in two categories: (i) the category of mobile protons (water and monomers protons) and (ii) the category of gelatin and polymer protons. Our model is constituted by two compartments: compartment 1 is formed by monomer protons and compartment 2 - by protons of growing or already formed polymers (see Fig. 1). The rest of the protons contribute to the formation of the signal but do not modify it.
Moreover, the protons in each of the compartments will contribute to the MRI signal in proportion to their densities. Therefore, this signal will consist of the signals emitted respectively by the protons of the water $\left(i_{\text {water }}\right)$, the protons of the gelatine $\left(i_{g}\right)$ and those of the two compartments. For a control sample (unirradiated gel), the MRI signal intensity is the sum of the signals emitted by the water protons, the gelatin protons and the monomer protons $P_{1}(0)$ (the initial number of monomer protons). Then, the MRI signal intensity for this sample is given by

$$
i_{0}=P_{1}(0) m_{0} \mathrm{e}^{-R_{20} \mathrm{TE}}+i_{\text {water }}+i_{g} .
$$

The number of protons of compartment 1 (protons of the monomers) is denoted by $P_{1}(t)$ and $P_{2}(t)$ is the number of protons of compartment 2 (protons of the polymers: growing or formed) with a probability of transfer per unit of time $p_{1}(t)$ proportional to the absorbed dose rate $\dot{D}(t)$. Thus,

$$
p_{1}(t)=k_{1} \dot{D}(t)
$$

and it is equivalent to the polymerization initiation probability.

The dose rate $\dot{D}(t)$ in the case of internal irradiation is given by [15]:

$$
\dot{D}(t)=\frac{C_{\mathrm{X}}}{m} a(t)
$$

with

$$
C_{\mathrm{X}}=k \sum_{i} n_{i} E_{i} \phi_{i}
$$

where $a(t)$ is the activity $[\mathrm{MBq}], n_{i}$ is the number of radiations having an energy $E_{i}[\mathrm{MeV}]$ emitted by the nuclear transition of the radioelement $\mathrm{X}, \phi_{i}$ is the fraction of emitted energy absorbed by the target, $m$ is the mass of the target $[\mathrm{kg}]$ and $k$ is a constant $[\mathrm{Gy} \mathrm{kg} /(\mathrm{MBq} \mathrm{s} \mathrm{MeV})]$.

Then, the absorbed dose at the time $t$ after the sample preparation is

$$
D(t)=\frac{C_{\mathrm{X}}}{m} \frac{a(0)}{\lambda_{p}}\left(1-\mathrm{e}^{-\lambda_{p} t}\right),
$$

where $\lambda_{p}=0.693 / T_{p}$ and $T_{p} \approx 8$ days is the physical period of iodine- 131 .

The model depicted in Fig. 1 can be accordingly described

$$
\frac{\mathrm{d} P_{1}(t)}{\mathrm{d} t}=-\frac{\mathrm{d} P_{2}(t)}{\mathrm{d} t}=-k_{1} \dot{D} P_{1}(t),
$$

then

$$
\left\{\begin{array}{l}
P_{1}(t)=P_{1}(0) \mathrm{e}^{-k_{1} \dot{D} t} \\
P_{2}(t)=P_{1}(0)\left(1-\mathrm{e}^{-k_{1} \dot{D} t}\right) .
\end{array}\right.
$$

At the time $\tau$ and the gel having received a dose $D(\tau)$, the relaxation rate increases and is given by

$$
\begin{aligned}
& R_{2}(\tau)=R_{20}+\alpha \frac{C_{\mathrm{X}}}{m} \frac{a(0)}{\lambda_{p}}\left(1-\mathrm{e}^{-\lambda_{p} \tau}\right)= \\
& R_{20}+\alpha \gamma(\tau),
\end{aligned}
$$

where $\alpha$ determines the change in the rate per unit dose. 
Then, the MRI signal intensity becomes

$$
\begin{aligned}
& i(\tau)=P_{1}(\tau) m_{0} \mathrm{e}^{-R_{20} \mathrm{TE}} \\
& \quad+P_{2}(\tau) m_{0} \mathrm{e}^{-\left(R_{20}+\alpha \gamma(\tau)\right) \mathrm{TE}} .
\end{aligned}
$$

For the MAGIC-iodine-131 gel, the signal intensity at the time $\tau$ is given by where

$$
i_{1}(\tau)=\zeta_{\text {mono }}(\tau)+\zeta_{\text {poly }}(\tau)+i_{\text {water }}+i_{g},
$$

$$
\left\{\begin{array}{l}
\zeta_{\text {mono }}(\tau)=P_{1}(0) m_{0} \mathrm{e}^{-k_{1} \gamma(\tau)} \mathrm{e}^{-R_{20} \mathrm{TE}} \\
\zeta_{\text {poly }}(\tau)=P_{1}(0) m_{0} \frac{1-\mathrm{e}^{-k_{1} \gamma(\tau)}}{\exp (\alpha \gamma(\tau) \mathrm{TE})} \mathrm{e}^{-R_{20} \mathrm{TE}}
\end{array}\right.
$$

In order to simplify the above expressions, one calculates

$$
\begin{aligned}
& \frac{i_{0}-i_{1}(\tau)}{\mathrm{e}^{-R_{20} \mathrm{TE}}}=P_{1}(0) m_{0}\left(1-\mathrm{e}^{-k_{1} \delta(\tau)}\right) \\
& \quad \times\left(1-\mathrm{e}^{-\mathrm{TE} \alpha \delta(\tau)}\right) .
\end{aligned}
$$

This result means that the number of polymers for each length will depend on the same parameters and the average of the proton relaxation rate $R_{2}=R_{20}+\alpha \bar{D}$ will change over time.

\section{Results}

In order to check the radioactivity homogeneity distribution in the samples, we have scanned them by scintigraphy. If $a_{j}$ is the activity contained in the sample $j$ and $i_{j}$ is the intensity of the scintigraphic signal emitted by this sample, then the signal intensity and activity are linked by a linear relation

$$
i_{j}=\alpha a_{j}+\beta
$$

where $\alpha$ and $\beta$ are two constants determined by the least squares method. The correlation coefficient is denoted by $r$ and $\beta$ represents the background noise. Curves presented in Fig. 2 give variations of the mean signal intensity measured by scintigraphy versus the activity of iodine-131 deposited in the MAGIC gel samples.

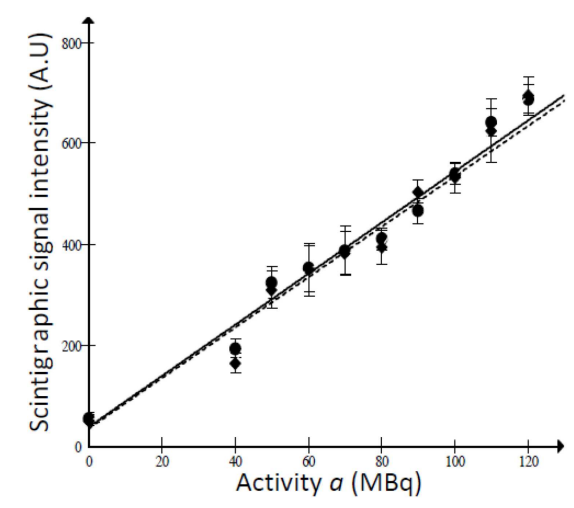

Fig. 2. Mean scintigraphic signal intensity (arbitrary unit) versus the activity $a$ deposited in the sample (average $\pm \sigma$ ). HAPG $=$ hour after preparation of the gel. 1 HAPG: $i(a)=5.50 a+$ 38.94, $r^{2}=0.972$; 3 HAPG: $i(a)=4.99 a+35.89$, $r^{2}=0.962$.

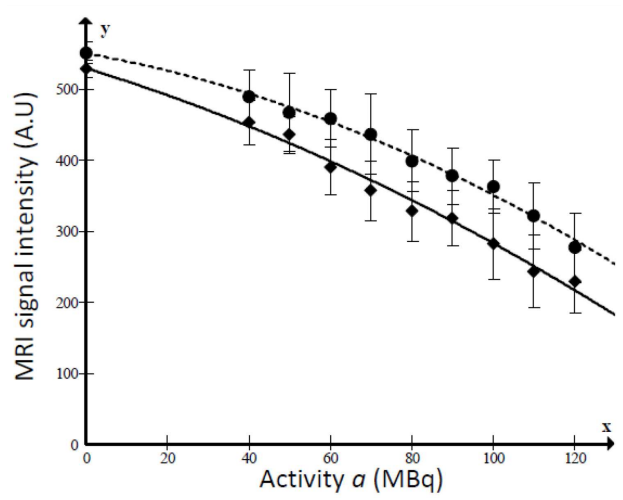

Fig. 3. Mean MRI signal intensity (arbitrary unit) versus the activity $a(0)$ deposited in samples (average $\pm \sigma$ ).

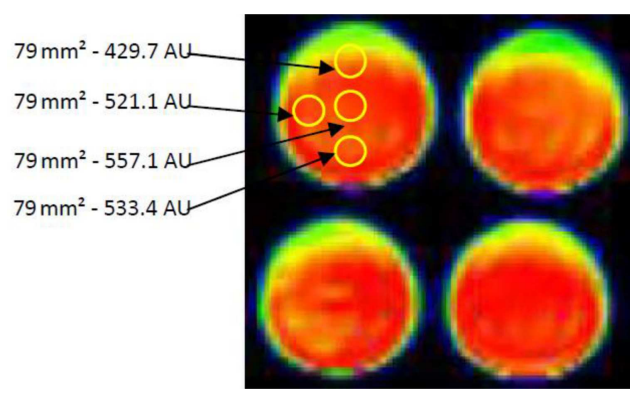

Fig. 4. Intensity MRI signal (arbitrary units) of four samples having received the same activity (80 MBq).

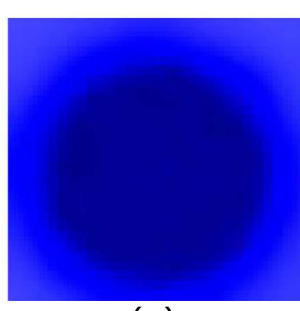

(a)

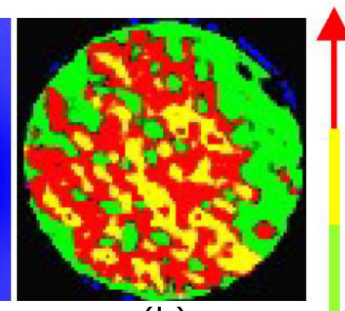

(b)
Fig. 5. NM (a) and MR (b) images of the same sample.

Furthermore, in order to study the rate of polymerization, we measured the intensity of the MRI signal emitted by each of the samples. We therefore considered the signal emitted by an axial section of the sample and studied the statistical dispersion of the signal between pixels. For each activity, five measures were taken. Curves in Fig. 3 give the average values, with the standard deviations of signal intensities measured for each activity (see Fig. 4).

The wide dispersion in signal strength observed between different regions of the same sample shows that this sample does not emit a homogeneous signal. This also means that the value of the relaxation time T2 varies from one point to another. Therefore, we manually segmented the different MR images by using a multilevel thresholding image segmentation [16] (see Fig. 5b). 


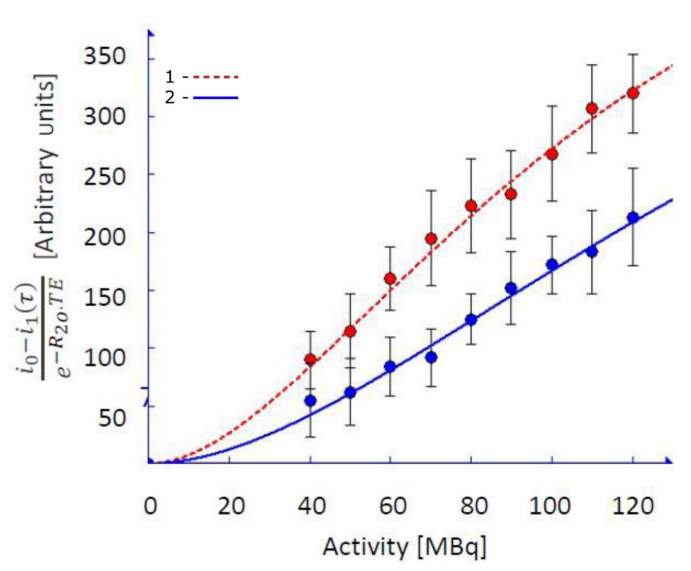

Fig. 6. Variations of the MRI signal intensity versus the deposited activity. Dashed line: 550(1 $\left.\mathrm{e}^{-3.28 \times 10^{-3} a}\right)\left(1-\mathrm{e}^{-1.90 \times 10^{-3} a}\right)$. Solid line: 550(1$\left.\mathrm{e}^{-1.24 \times 10^{-4} a}\right)\left(1-\mathrm{e}^{-2.14 \times 10^{-3} a}\right)$ (experimental values and fitted curves from (10)).

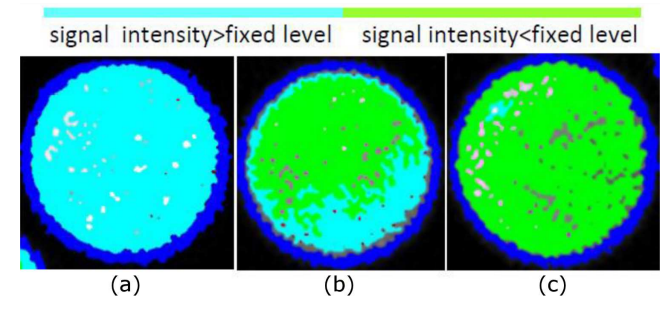

Fig. 7. MR images of the same sample segmented by using multilevel thresholding. (a) Without activity, (b) 72 HAPG, and (c) 120 HAPG.

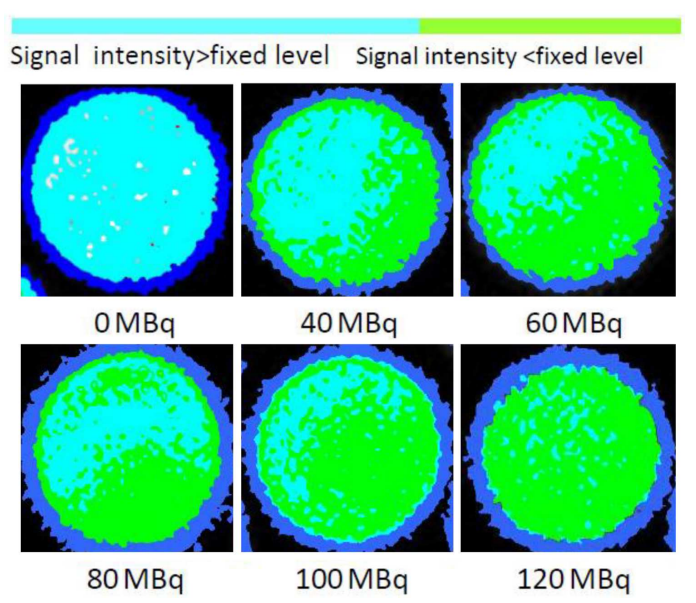

Fig. 8. MR images segmented by using multilevel thresholding obtained for different deposited activities.

In order to validate the proposed mathematical model, the experimental values were fitted using (10) (see Fig. 6). Furthermore, the MR images of samples were segmented into two classes by using a multilevel thresholding image segmentation [16] (see Figs. 7 and 8).

\section{Discussion}

Dosimetry studies are generally difficult to perform in vectorized internal radiotherapy. By measuring the variations of the MRI signal of the irradiated gels [17], we studied the homogeneity of the radio-induced polymerization. Scintigraphy and MRI images showed that a homogeneous distribution of 131I activity does not necessarily imply isotropic polymerization. Indeed, Fig. 3b shows that the polymerization seems not to be isotropic despite a homogeneous distribution of radioactivity (Fig. 5a). This phenomenon is probably linked to the fact that iodine-131 emits beta particles with different energies and the length of the polymers does not increase with the same speed for all the polymers in formation. Moreover, this result can be explained by the fact that the conversion curves of methacrylic acid are linear and the resulting polymer does not appear to have a stereoregular structure.

The functioning of any polymer gel system is the radiation-induced polymerization of the monomer species present in the gel. Traditionally, radiation induces polymerization of a monomer and a cross linker. In single monomer systems, several factors affect the rate of polymerization or the rate of monomer consumption. The observed heterogeneous polymer structure can be attributed to a possible increase in the termination directly related to the initial monomer concentration.

However, these first results suggest that additional measurements must be carried out in order to study with precision the polymerization process and its influence on the MRI signal of the MAGIC gel dosimeter.

The values of the calculated standard deviations (Fig. 3) show a large dispersion of the signal intensities on the same sample. This dispersion is due to the fact that the irradiation is carried out continuously with a decreasing dose rate. As a result, the polymerization cannot evolve in the same way at all points of the sample.

Images in Fig. 5 were made on the same sample, before the introduction of radioactivity (control sample), $72 \mathrm{~h}$ and $120 \mathrm{~h}$, respectively, after the introduction of iodine-131. These images were segmented manually to highlight that two distinct areas give different intensities of the MRI signal. Also, despite a uniform distribution of radioactivity in the sample (verified by scintigraphic images), the polymerization does not appear to have constant kinetics in the sample. This result is in agreement with our hypotheses made to build the proposed mathematical model (Fig. 1). Furthermore, the image in Fig. 5 shows that the polymer compartment contains several sub-compartments emitting signals of different intensities which justifies the hypothesis according to which, at a given instant $t$, the length of the polymers is not the same. 
The curves in Fig. 5 show that the values of the intensity of the MRI signal can be fitted by the equations established from the proposed model. However, the initial equations do not take into account the fact that the relation rate $R$ varies with time. It is then necessary to establish a mathematical relationship which accounts for these variations.

We have also noted that the $\frac{k_{1}}{a \mathrm{TE}}$ ratio varies as a function of time. In fact, it is 0.748 and $0.580,72 \mathrm{~h}$ and $120 \mathrm{~h}$, respectively, after the preparation of the samples. Since TE is fixed by the measurement conditions, the varying values are those of $k_{1}$ and $a$. It seems that these two parameters are modified by the presence of polymers which increases with time. However, it is necessary to consider more complex measurements to confirm variations in the ratio of these two parameters and verify that these variations are statistically significant.

\section{Conclusion}

A polymer gel is a highly reliable $3 \mathrm{D}$ dosimeter in vectorized internal radiotherapy. The basic principle is to measure the variations of the intensity of the MRI signal emitted by this dosimeter. It has been shown that these variations in the MRI signal are directly linked to the kinetics of the radiationinduced polymerization. However, our measurements performed on samples receiving internal irradiation suggest that this kinetics is neither homogeneous nor isotropic. On this basis, we have established a mathematical model which compartmentalizes the different species of protons that participate in the modification of the MRI signal. From this model, the mathematical equation of the MRI signal emitted by the irradiated gel, as a function of the activity initially present in the samples, was established. In addition, we have shown that the equation describing the variations in the relaxation rate $\mathrm{R} 2$ is not a linear function of the absorbed dose but rather a function with an exponential component. Therefore, our results seem to be in contradiction with the data in the literature but in reality they complement them because they suggest correcting the value of the absorbed dose taking into account the calibration equation R2 (D) that we established.

Moreover, our experimental results show that it is also necessary to introduce the probabilistic aspect of the radiation-induced polymerization of polymer gels (polymerization growth, early termination of the polymerization, etc.) into the equations describing the proposed mathematical model. This is precisely the work currently carried out by our team with the aim to establish the mathematical equations of different stages of radiation-induced polymerization in order to take them into account in the modelling.

Finally, the obtained results can serve as a basis for the development of a new approach in the determination of the absorbed dose from the calibration curves.

\section{References}

[1] S. Becker, S. Laffont, F. Vitry et al., Nucl. Med. Commun. 29, 815 (2008).

[2] J. Gear, C. Chiesa, M. Lassmann, P.M. Gabiña, J. Tran-Gia, C. Stokke, G. Flux, EJNMMI Phys. 7, 15 (2020).

[3] L. Strigari, M. Konijnenberg, C. Chiesa, M. Bardies, Y. Du, K.S. Gleisner, M. Lassmann, G. Flux, Eur. J. Nucl. Med. Mol. Imaging 41, 1976 (2014).

[4] W.S. Snyder, M.R. Ford, G.G. Warner, H.L. Fisher, J. Nucl. Med. 10, 5 (1969).

[5] R. Loevinger, M. Berman, MIRD Pamphlet No. 1, Society of Nuclear Medicine, New York 1976.

[6] P.M. Fong, D.C. Keil, M.D. Does, J.C. Gore, Phys. Med. Biol. 46, 3105 (2001).

[7] M. Keshtar, A. Takavar, M.H. Zahmatkesh, A.R. Montazerabadi, J. Biomed. Phys. Eng. 7, 299 (2017).

[8] M. Ferry, Y. Ngono-Ravache, C. AymesChodur, M.C. Clochard, X. Coqueret, L. Ortella, E. Pellizzi, S. Rouif, S. Esnouf, in: Reference Module in Materials Science and Materials Engineering, Elsevier, Amsterdam 2016.

[9] G. Odian, Principles of Polymerization, 4th ed., Wiley, New York 2004.

[10] C. Baldock, Y. De Deene, S. Doran, G. Ibbott, A. Jirasek, M. Lepage, K.B. McAuley, M. Oldham, L.J. Schreiner, Phys. Med. Biol. 55, 1 (2010).

[11] F. Courbon, P. Love, S. Chittenden, G. Flux, P. Ravel, G. Cook, Cancer Biother. Radiopharmaceut. 2, 427 (2006).

[12] W.Y. Chiu, G.M. Carratt, D.S. Soong, Macromolecules 16, 348 (1983).

[13] K. Matyjaszewski, T.P. Davis, Handbook of Free Radical Polymerization, Wiley, New York 2003.

[14] J.W.T. Spinks, R.J. Woods, An Introduction to Radiation Chemistry, 3rd ed., Wiley, New York 1990.

[15] W.E. Bolch, K.F. Eckerman, G. Sgouros, R. Thomas, J. Nucl. Med. 50, 477 (2009).

[16] S. Pare, A. Kumar, G.K. Singh, V. Bajaj, Iran. J. Sci. Technol. Trans. Electr. Eng. 44, 1 (2020).

[17] M.J. Maryanski, J.C. Gore, R.P. Kennan, R.J. Schulz, Magn. Reson. Imaging 11, 253 (1993). 